\title{
Revealing the nature of radar reflections in ice: DEP-based FDTD forward modeling
}

\author{
Olaf Eisen, Frank Wilhelms, Uwe Nixdorf, and Heinrich Miller \\ Alfred-Wegener-Institut für Polar- und Meeresforschung Bremerhaven, Bremerhaven, Germany \\ Received 7 October 2002; revised 25 November 2002; accepted 15 January 2003; published XX Month 2003.
}

[1] Successful simulation of ground penetrating radar (GPR) traces in polar ice is achieved by numerical finitedifference time-domain (FDTD) forward modeling. Properties of the modeled medium are taken from high resolution dielectric profiling (DEP) of the upper $100 \mathrm{~m}$ of an ice core from Dronning Maud Land, Antarctica. The GPR reference trace is calculated from stacking of a normal moveout corrected common-midpoint survey, carried out near the borehole location. The excellent agreement of synthetic and GPR-based results demonstrates the capability of FDTD models to reproduce radargrams from ice core properties for interpretation of radio echo sounding data, and emphasizes the exploitation of radar data for improved interpretations of glaciological climate proxys. In addition to presenting modeling results, we perform sensitivity experiments to investigate the nature and origin of radar reflection in ice, discuss reasons for the failure of modeling studies in the past, and indicate new approaches. INDEX TERMS: 0644 Electromagnetics: Numerical methods; 0689 Electromagnetics: Wave propagation (4275); 1863 Hydrology: Snow and ice (1827); 1827 Hydrology: Glaciology (1863); 9310 Information Related to Geographic Region: Antarctica. Citation: Eisen, O., F. Wilhelms, U. Nixdorf, and H. Miller, Revealing the nature of radar reflections in ice: DEP-based FDTD forward modeling, Geophys. Res. Lett., 30(0), XXXX, doi:10.1029/ 2002GL016403, 2003.

\section{Introduction}

[2] Radio echo sounding has become a standard tool for glaciological applications to determine ice thickness, internal structure, basal properties, accumulation rates, and ice dynamics [e.g., Robin et al., 1969; Oswald and Robin, 1973; Hempel and Thyssen, 1992; Arcone et al., 1995; Corr et al., 2002]. The basic processes are reasonably well understood, i.e. partial reflection of the propagating electromagnetic (EM) waves at dielectric discontinuities. Most applications related to internal structures, however, still depend on assumptions and require further information to enable unambiguous data interpretations.

[3] Several studies have demonstrated the importance of density in the upper part of the ice sheet and the connection of volcanic events with deeper internal reflection horizons [e.g., Millar, 1981; Bogorodsky et al., 1985; Siegert, 1999; Hempel et al., 2000]. However, calculation of corresponding synthetic radargrams, based on density and conductivity profiles, only had very limited success [Miners et al., 1997]. Although utilization of the complex dielectric constant from

Copyright 2003 by the American Geophysical Union. 0094-8276/03/2002GL016403\$05.00 dielectric profiling (DEP) in recent studies has partly advanced the agreement between synthetic and measured radargrams [Kohler et al., 2003; Eisen et al., 2003], further improvements are still necessary.

[4] This study demonstrates that problems related to the limited representation of wave phenomena in earlier models (for detailed summaries see Miners [1998]; Hildebrand [1996]) and large lateral inhomogeneities of physical and chemical properties of ice (L. Karlöf, Norwegian Polar Institute, personal communication, 2002) can be overcome by adopting established numerical modeling and radar processing techniques from other fields. We present a successful reproduction of measured ground penetrating radar (GPR) data by a finite-difference time-domain (FDTD) modeling approach based on ice core DEP data. The successful comparison of the modeled radargram with processed radar data validates the underlying modeling approach and facilitates sensitivity studies to proof the power of FDTD modeling as a tool for physical-chemical studies on the nature of radar reflections in ice.

\section{DEP}

[5] Modeling of EM wave propagation requires knowledge of the physical structure of the medium of interest. The most accurate prescription of medium properties is provided by the vertical distribution of the dielectric constant from DEP measurements along ice cores [Moore and Paren, 1987].

[6] The improved DEP device developed by Wilhelms et al. [1998], and further refined by Wilhelms [2000], allows the simultaneous measurement of both components of $\varepsilon=\varepsilon^{\prime}$ $-i \varepsilon^{\prime \prime}=\varepsilon^{\prime}-i \sigma\left(\varepsilon_{0} \omega\right)^{-1}$, where the real part $\varepsilon^{\prime}$ is the ordinary relative permittivity of the medium; the imaginary part $\varepsilon^{\prime \prime}$ is the dielectric loss factor. The latter can be expressed as a function of conductivity $\sigma$; angular frequency $\omega$; and permittivity of vacuum $\varepsilon_{0}$.

[7] The ice core B32 was retrieved in 1997/98 during the EPICA pre-site survey in Dronning Maud Land, Antarctica [Oerter et al., 2000], and profiled at a frequency of $250 \mathrm{kHz}$ in $\Delta z=5 \mathrm{~mm}$ increments (Figure 1). Each component has a systematic accuracy of about $1 \%$ and a statistical error of $0.1 \%$. Sections with peaks of low $\varepsilon^{\prime}$, caused by cracks in parts of poor core quality, were removed in both $\varepsilon$-components, if the actual value differed from the $2.5 \mathrm{~m}$ running mean bymore than one standard deviation of the same window. In total, $4.5 \%$ of the final $\varepsilon$-depth series were missing or had to be rejected.

\section{FDTD Modeling}

[8] Advantages of the FDTD technique are the implicit inclusion of EM wave phenomena occuring during prop- 
(a)

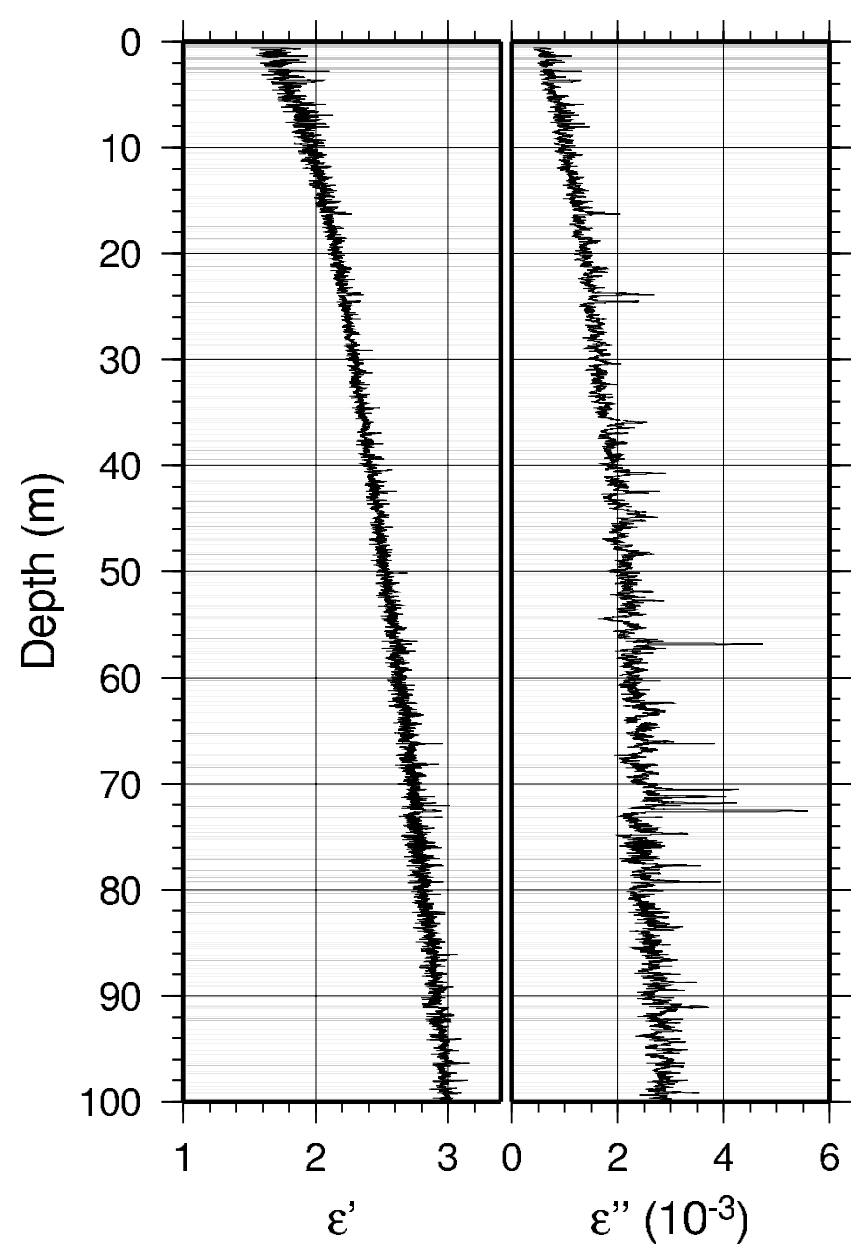

Figure 1. Dielectric properties of ice core B32 as a function of depth. (a) Ordinary relative permittivity $\varepsilon^{\prime}$; (b) dielectric loss factor $\varepsilon^{\prime \prime}$, measured at $250 \mathrm{kHz}$ and scaled to $200 \mathrm{MHz}$. The underlain gray narrow horizontal bars indicated gaps in the DEP data record.

agation, like multiple reflections, interferences, absorption, geometrical spreading, ray focusing, phase shifts, etc. Our numerical model is based on a FDTD set of Maxwell's curl equations [Yee, 1966], implemented on a staggered grid with absorbing boundary conditions. (Taflove [1995] provides extensive discussions on physical interpretations, implementation approaches, and other applications.) Results of this study are obtained with a 2D version of the model, with the transmitter (TX) being represented by an infinite electric line source perpendicular to the $(x, z)$ model space ( $E_{y}$-polarized source signal), thereby decoupling the transversal electric (TE) and magnetic modes (TM) [Hohmann, 1988]. Duration and shape of the source wavelet are determined from the air wave of the $200 \mathrm{MHz}$ commonmidpoint (CMP) radargram described below, interpolated onto equidistant time increments $\Delta t$ using an Akima spline (details on the wavelet can be found in Eisen et al. [2003]). The DEP data are linearly interpolated onto the cartesian space grid, assuming lateral homogeneity in ice and air, with $\varepsilon_{\text {air }}=1$ and $\sigma_{\text {air }}=0$.
[9] The parallelized model was implemented on a NEC SX-6 supercomputer at the Deutsches Klimarechenzentrum (DKRZ, Hamburg, Germany). Model dimensions for all runs are $10 \mathrm{~m}$ in horizontal direction $(x \in[-5 \mathrm{~m}, 5 \mathrm{~m}])$, and $120 \mathrm{~m}$ in verticaldirection $(z \in[-20 \mathrm{~m}, 100 \mathrm{~m}])$, with an isotropic space increment $\Delta=0.01 \mathrm{~m}$ and a time increment $\Delta t=0.01 \mathrm{~ns}$, thus providing high resolution of the source pulse, satisfying the stability criterion, and reducing numerical dispersion [Taflove, 1995]. Further decreasing space and time increments for testing purposes did hardly change results, confirming that numerical diffusion can be neglected at this frequency and level of DEP resolution. The TX is positioned at $(x, z)=(-0.25 \mathrm{~m},-0.05 \mathrm{~m})$, the receiver $(\mathrm{RX})$ at $(x, z)=(0.25 \mathrm{~m},-0.05 \mathrm{~m})$, representing the survey geometry of the first CMP trace. Simulation of 1000 ns of wave propagation with this parameter set resulted in the synthetic radargram $S_{F D}$. The computation took less than $1 \mathrm{~h}$, required 1.5 GBytes RAM memory, and $7 \mathrm{~h}$ of CPU time at 3.7 GFlops on 8 dedicated CPUs.

\section{Radar Reference Data}

[10] Comparisons of modeled radargrams with measured radar data require suitable reference traces. These can either be derived from single point, common-offset or CMP radar surveys. Whereas the first two survey setups are carried out at a fixed TX-RX distance, stationary in the first case and moving across the surface in the second, the CMP recording technique, adapted from seismic data acquisition, provides multifold coverage at several nonzero-offsets, thereby improving SNR and yielding subsurface velocity information [Garotta and Michon, 1967; Yilmaz, 1987].

[11] The CMP survey was recorded $\sim 100 \mathrm{~m}$ south of the borehole B32 in 1998/99 with a commercial RAMAC GPR device (Mal Geoscience, Sweden), operated with two unshielded $200 \mathrm{MHz}$ dipole antennae. Starting with a minimal TX-RX offset of $1.0 \mathrm{~m}, 139$ traces were recorded at $0.5 \mathrm{~m}$ offset increments, applying 512-fold vertical stacking to each trace. The CMP-based velocity model [Eisen et al., 2002] is used to apply a normal moveout (NMO) correction to each trace. Subsequent stacking of all NMO corrected traces results in one single reference trace, $S_{C M P}$, used for validation of $S_{F D}$.

\section{Synthetic Versus Reference Trace}

[12] Before carrying out the comparison of modeled and measured radargrams three problems need to be considered: first, a separation between drilling location and CMP survey of some $100 \mathrm{~m}$; second, one year of accumulation between ice core retrieval and GPR survey; and third, simulation of a $3 \mathrm{D}$ wave propagation process with a $2 \mathrm{D}$ model. These differences are partly taken care of by linearly correcting the CMP two-way traveltime (TWT) to the 1998 surface-depth structure at the borehole and applying a 200 ns automatic gain control filter to both traces, also correcting for antenna gain and system properties. The comparison is limited to the interval 100-1000 ns TWT, both to avoid interference of the air and ground wave with internal reflection hyperbolae in the CMP data, and to reduce the influence of bad core data at greater depth. 


\section{CMP CMP FD FD}

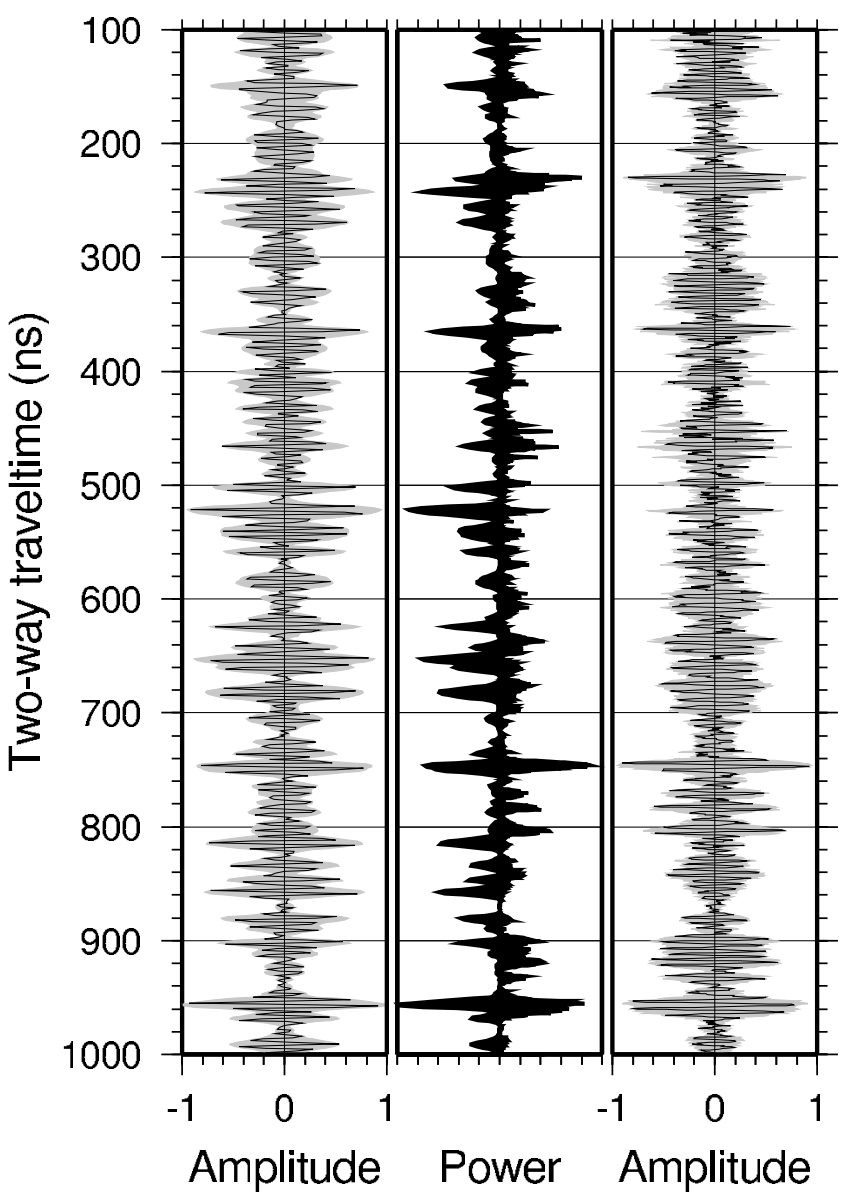

Figure 2. Comparison of CMP and FD trace amplitude and power as a function of two-way traveltime (TWT). The left panel shows the gain corrected CMP-based trace $S_{C M P}$ in wiggle format, underlain by the trace envelope (displayed in positive and negative axis directions). The right panel shows the corresponding FD-based trace $S_{F D}$. The middle panel shows the power of both traces, calculated from the squared envelopes, with the power of $S_{C M P}$ increasing to the left, and $S_{F D}$ increasing to the right. All displays are normalized to the maximum magnitude in the interval $100-$ 1000 ns.

[13] Comparing both radargrams indicates similarities, but also demonstrates their different origin (Figure 2). Whereas most signals in $S_{C M P}$ are distinct events, fewer reflections in $S_{F D}$ clearly rise above background noise. This is, however, not surprising, as a noise-reducing processing step similar to CMP stacking is missing for the modeled trace, i.e. the noise inherent to the DEP data is still present in $S_{F D}$, with little numerical diffusion added, resulting in a lower SNR than for $S_{C M P}$.

[14] Although consideration of the power distribution instead of amplitude-phase relations of return signals comes along with a loss of phase information, it results in a more robust quantity for comparison and applications like tracking of internal reflection horizons, as strong reflections are emphasized by reduced noise level. The distributions of major reflections show an excellent agreement. Numerous peaks occur in both power radargrams, several of which are of comparable magnitude, the most obvious ones at 220 , 360, 740 and 950 ns TWT. Despite the lower SNR for $S_{F D}$, several events show good agreement with $S_{C M P}$ not only in their onset and magnitude, like the reflections at $340 \mathrm{~ns}$, but also in the phase structure, e.g. events at 740 and $950 \mathrm{~ns}$. In some cases the reflection pattern is comparable, but the magnitudes differ, like the double peaks around $240 \mathrm{~ns}$ in either radargram with reversed order of reflection strength, or the 500-520 ns sequence.

[15] Different schemes were applied for evaluating and correcting the DEP data set. The results emphasize that the $\varepsilon$-depth distribution is the most important factor for reproducing survey radar data. Missing or wrong DEP data sections have more severe influence on the synthetic trace than slightly different source wavelets, numerical approximations, or model geometry. In particular, agreement of the phase structure of reflections originating from only partly consolidated firn cannot be expected in general, as the lateral dielectric variations of small scale features like sastrugis occur within the first Fresnel zone, implying interference of the reflected elementary wave trains. A lateral homogeneous $\varepsilon$-distribution does not reproduce these variations.

[16] An objective measure of the agreement of traces is important for comparing different modeling approaches or judging sensitivity studies. One example is the correlation coefficient of trace power distributions, which results as 0.43 . The correlation coefficients of trace amplitudes is less suitable for evaluating the agreement, indicated by the low value of 0.13 , as small differences in timing of phase shifts of single events can completely destroy the correlation.

\section{Sensitivity Studies}

[17] The good agreement between modeled and measured radargrams validates the modeling approach and enables the numerical investigation of the physical origin of reflections. To this end we perform two sensitivity experiments. We calculate a synthetic radargram, $S_{I}$, from a smoothed distribution of $\varepsilon^{\prime}$, filtered with a $20 \mathrm{~m}$ boxcar running mean, and unchanged $\varepsilon^{\prime \prime}$. The opposite filtering is carried out for a second experiment, i.e. only $\varepsilon^{\prime \prime}$ is filtered, resulting in $S_{I I}$. Whereas the amplitudes and phases of reflections in $S_{I}$ are quite different from those of the original synthetic radargram $S_{F D}, S_{I I}$ is very similar (Figure 3).

[18] This has several implications. The second study demonstrates that the reflection structures of $S_{F D}$, and because of their good agreement also of $S_{C M P}$, are almost solely determined by permittivity. Reflections caused by changes in conductivity are 1-2 orders of magnitude smaller than those caused by variations in permittivity in the considered depth range, as seen from $S_{I}$. Moreover, changes in conductivity are also too small to have a considerable influence on the phase structure of reflection signals.

[19] The results confirm earlier findings [Moore, 1988; Hildebrand, 1996; Miners, 1998] that the reflection coefficient in the upper hundreds of meters is dominated by changes in permittivity and that conductivity changes are negligible. An interesting feature is the occurrence of three 


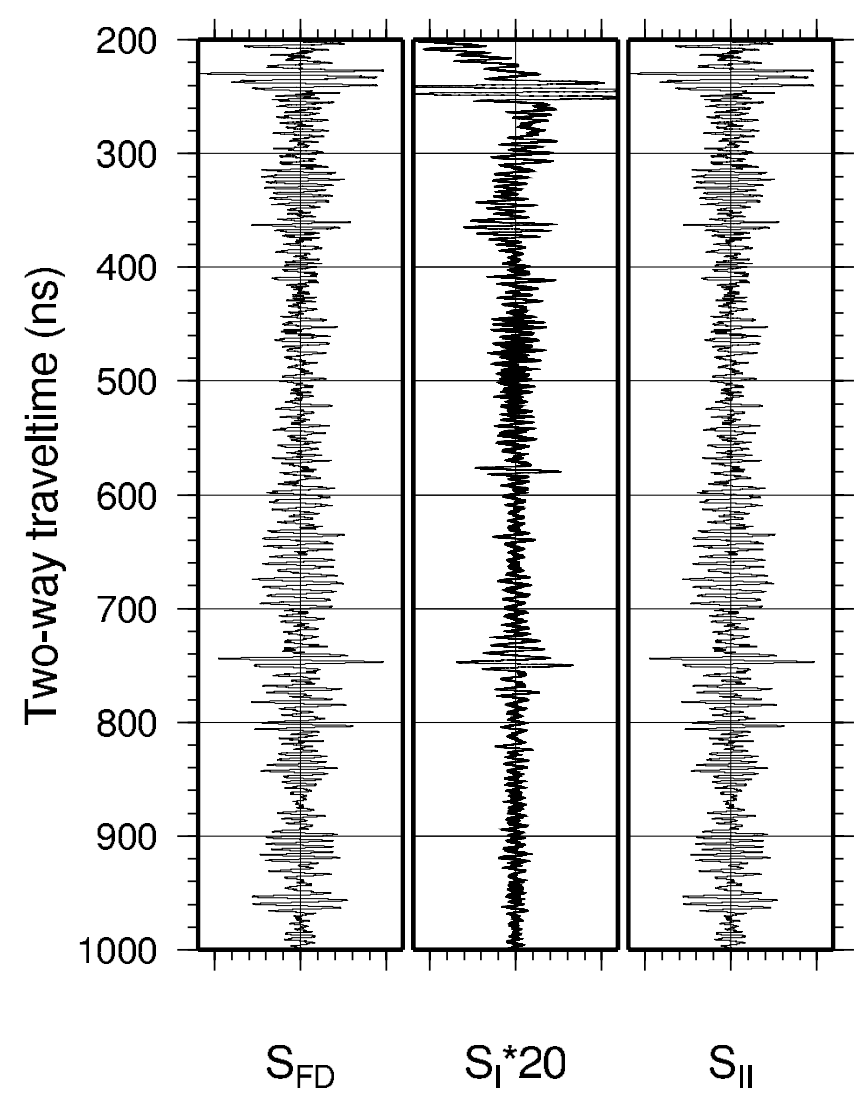

Figure 3. Comparison of the original FD-trace $S_{F D}$ with the two results of the sensitivity studies. Note that the amplitude of $S_{I}$ is multiplied by a factor of 20 .

distinct reflections in $S_{I}$ at the same depth as in $S_{I I}$, that also show comparable amplitude ratios (at 240, 360 and 740 ns TWT). This implies that the change in permittivity of the ice core record, responsible for the reflections in $S_{I I}$ and $S_{F D}$, is accompanied by a change in conductivity, or vice versa. Possible explanations are: dielectric mixing of the complex $\varepsilon$ of the air-snow mixture according to the general cubical power law proposed by Landau and Lifschitz [1982] in the 1940 s, and investigated by means of DEP by Wilhelms [2000]; direct influence of chemical impurities on the permittivity [Fujita et al., 2000]; or a common formation process, as found in other radar data [Eisen et al., 2003].

\section{Summary}

[20] This study demonstrates that successful modeling of GPR data in polar ice can be achieved by means of the DEP-based FDTD technique. Problems of other approaches, like lacking consideration of important physical processes in convolution models or utilization of a density-based permittivity, are overcome by their implicit inclusion in the governing equations and provision of an accurate picture of the complex physical properties of the subsurface by high resolution in-situ DEP data, respectively.

[21] Lateral inhomogeneities of the medium and gaps in the DEP data are likely the main reasons for remaining discrepancies. On the GPR side, reduction of noise can be achieved by analysis of CMP surveys. For modeling, improved solutions with lower noise level could be obtained by either stacking ice core profiles from neighboring cores or simulating CMP-like survey setups with subsequent processing, unfortunately requiring a huge amount of computer time and memory. Apart from limitations set by the inhomogeneous nature of the ice pack, exact matching of measured and synthetic radargrams requires model extension to $3 \mathrm{D}$ and accurate representation of antennae and system properties.

[22] Nevertheless, the present success already encourages the study of electromagnetic reflection phenomena in deeper parts of the ice sheets by forward modeling, the most important ones being the transition from permittivity to conductivity dominated reflections and the influence of ice fabric on signal properties. The new EPICA ice core from Dronning Maud Land, in conjunction with airborne radar data, provides a promising data set to achieve these goals in the near future.

[23] Acknowledgments. The original version of the FDTD model was developed by Volker Mayer and Jupp Sandmeier as a module for the commercial GPR analysis PC software Reflex (Sandmeier Software, Karlsruhe, Germany). They gave helpful advice for decoupling the module from the PC software for our special purposes. Debugging and optimization greatly profited from support by Stephan Frickenhaus, AWI computing centre, and Klaus Ketelsen, DKRZ. The paper benefited from the comments by Jack Kohler and Richard Hindmarsh (reviewers), the contribution from James Famiglietti (scientific editor) is greatly acknowledged. Preparation of this work was supported by the Deutsche Forschungsgemeinschaft grant Ni493/1 and two scholarships of the Studienstiftung des Deutschen Volkes. This work is a contribution to the "European Project for Ice Coring in Antarctica" (EPICA), a joint ESF (European Science Foundation)/EC scientific programme, funded by the European Commission and by national contributions from Belgium, Denmark, France, Germany, Italy, the Netherlands, Norway, Sweden, Switzerland and the United Kingdom. This is EPICA publication no. 55.

\section{References}

Arcone, S., D. Lawson, and A. Delaney, Short-pulse wavelet recovery and resolution of dielectric contrasts within englacial and basal ice of Matanuska Glacier, Alaska, U.S.A., J. Glaciol., 41, 68-86, 1995.

Bogorodsky, V., C. Bentley, and P. Gudmandsen, Radioglaciology, D. Reidel, Norwell, Mass., 1985.

Corr, H. F. J., A. Jenkins, K. W. Nicholls, and C. S. M. Doake, Precise measurement of changes in ice-shelf thickness by phase-sensitive radar to determine basal melt rates, Geophys. Res. Lett., 29(8), 1232, doi:10.1029/ 2001GL014618, 2002.

Eisen, O., U. Nixdorf, F. Wilhelms, and H. Miller, Electromagnetic wave speed in polar ice: Validation of the CMP technique with high resolution DEP and $\gamma$-density measurements, Ann. Glaciol., 34, 150-156, 2002.

Eisen, O., F. Wilhelms, U. Nixdorf, and H. Miller, Identifying isochrones in GPR profiles from DEP-based forward modelling, Ann. Glaciol., 37, in press, 2003

Fujita, S., T. Matsuoka, T. Ishida, K. Matsuoka, and S. Mae, A summary of the complex dielectric permittivity of ice in the megahertz range and its application for radar sounding of polar ice sheets, in The Physics of Ice Core Records, 1st ed., edited by T. Hondoh, pp. 185-212, Hokkaido Univ. Press, Sapporo, Jpn., 2000.

Garotta, R., and D. Michon, Continuous analysis of the velocity function and the move out corrections, Geophys. Prospect., 15, 584-597, 1967.

Hempel, L., and F. Thyssen, Deep Radio Echo Soundings in the vicinity of GRIP and GISP2 Drill Sites, Greenland, Polarforschung, 62, 11-16, 1992.

Hempel, L., F. Thyssen, N. Gundestrup, H. B. Clausen, and H. Miller, A comparison of radio-echo sounding data and electrical conductivity of the GRIP ice core, J. Glaciol., 46, 369-374, 2000.

Hildebrand, A., Untersuchung der Laufzeit- und Amplitudenverhalten elektromagnetischer Impulse bei glaziologischen Radarmessungen, Ph.D. thesis, Westfälische Wilhelms-Uuniv. Münster, Münster, Germany, 1996.

Hohmann, G. W., Numerical modeling for electromagnetic methods of geophysics, in Electromagnetic Methods in Applied Geophysics, Geophys. Monogr. Ser., vol. 1, edited by M. N. Nabighian, pp. 313-364, Soc. of Explor. Geophys., 1988.

Kohler, J., J. C. Moore, and E. Isaksson, Comparison of modelled and observed responses of a glacier snowpack to ground-penetrating radar, Ann. Glaciol., 37, in press, 2003. 
Landau, L. D., and E. M. Lifschitz, Elektrodynamik der Kontinua, Lehrbuch der Theor, Phys., vol. VIII, Akademie, Berlin, 1982.

Millar, D. H. H., Radio echo layering in polar ice sheets and past volcanic activity, Nature, 292, 441-443, 1981.

Miners, W. D., Electromagnetic reflections inside ice sheets, Ph.D. thesis, Open Univ., 1998.

Miners, W., A. Hildebrand, S. Gerland, N. Blindow, D. Steinhage, and E. Wolff, Forward modelling of the internal layers in radio echo sounding using electrical and density measurements from ice cores, J. Phys. Chem. $B, 101,6201-6204,1997$.

Moore, J., Dielectric variability of a $130 \mathrm{~m}$ Antarctic ice core: implications for radar sounding, Ann. Glaciol., 11, 95-99, 1988.

Moore, J., and J. Paren, New technique for dielectric logging of Antarctic ice cores, J. Phys. Colloque, 48, 155-160, 1987.

Oerter, H., F. Wilhelms, F. Jung-Rothenhäusler, F. Göktas, H. Miller, W. Graf, and S. Sommer, Accumulation rates in Dronning Maud Land as revealed by dielectrical-profiling measurements at shallow firn cores, Ann. Glaciol., 30, 27-34, 2000.

Oswald, G. K. A., and G. D. Q. Robin, Lakes beneath the Antarctic ice sheet, Nature, 245, 251-254, 1973.

Robin, G. D. Q., S. Evans, and J. T. Bailey, Interpretation of radio echo sounding in polar ice sheets, Philos. Trans. R. Soc. London, Ser. A, 146 $437-505,1969$.
Siegert, M., On the origin, nature and uses of Antarctic ice-sheet radio-echo layering, Prog. Phys. Geogr., 23, 159-179, 1999.

Taflove, A., Computational Electrodynamics: The Finite-Difference TimeDomain Method, Artech House, Norwood, Mass., 1995.

Wilhelms, F., Measurement of Dielectric Properties of Polar Ice Cores (in German), Ber. Polarforschung, vol. 367, Alfred-Wegener-Inst. für Polarund Meeresforschung, Bremerhaven, Germany, 2000.

Wilhelms, F., J. Kipfstuhl, H. Miller, K. Heinloth, and J. Firestone, Precise dielectric profiling of ice cores: A new device with improved guarding and its theory, J. Glaciol., 44, 171-174, 1998.

Yee, K. S., Numerical solution of initial boundary value problems involving Maxwell's equations in isotropic media, IEEE Trans. Antennas Propag., 14, 302-307, 1966.

Yilmaz, O., Seismic Data Processing, Investig. Geophys. vol. 2, Soc. of Explor. Geophys., 1987.

O. Eisen, H. Miller, U. Nixdorf, and F. Wilhelms, Alfred-Wegener-Institut für Polar- und Meeresforschung, Postfach 120161, 27515 Bremerhaven, Germany. (oeisen@awi-bremerhaven.de) 